\title{
ANÁLISIS MINERALÓGICO \\ Y MULTIELEMENTAL DE LA CENIZA VOLCÁNICA, PRODUCTO DE LA ERUPCIÓN DEL COTOPAXI EN 2015, POR DIFRACCIÓN DE RAYOS X (XRD) Y ESPECTROMETRÍA DE MASAS CON PLASMA ACOPLADO INDUCTIVAMENTE (ICP-MS) Y SUS POSIBLES APLICACIONES E IMPACTOS
}

MINERALOGICAL AND MULTI-ELEMENTAL ANALYSIS OF THE VOLCANIC ASH, PRODUCT OF THE COTOPAXI ERUPTION ON 2015 BY X-RAY DIFFRACTION (XRD) AND INDUCTIVELY COUPLED PLASMA MASS SPECTROMETRY (ICP-MS) AND ITS POSSIBLE APPLICATIONS AND IMPACTS

Esteban Sánchez, ${ }^{1}$ Fátima Mejía, ${ }^{2}$ Gabriel Vizcaíno ${ }^{3}$ e Isabel Cipriani-Ávila ${ }^{1}$ Recibido: 25 septiembre 2017 / Aceptado: 28 noviembre 2017

Palabras claves: ceniza volcánica, efectos, ICP-MS, volcán Cotopaxi, XRD. Keywords: Cotopaxi volcano, effects, ICP-MS, volcanic ash, XRD

\footnotetext{
1 Pontificia Universidad Católica del Ecuador, Facultad de Ciencias Exactas y Naturales, Escuela de

Ciencias Químicas, Quito, Ecuador (esanchez8000@gmail.com; isabel_cipriani@yahoo.com).

2 Laboratorio Gruentec Cia. Ltda., Quito, Ecuador (fatimamejia88@hotmail.es).

3 PROINSTRA S.A., Quito, Ecuador (gabrielvizcaino@proinstra.com).
} 


\section{RESUMEN}

Este estudio tuvo como objetivo principal el análisis mineralógico y multielemental de la ceniza volcánica producto de la erupción del volcán Cotopaxi en agosto de 2015; con el fin de determinar posibles aplicaciones e impactos. Las técnicas de difracción de rayos X (XRD) y espectrometría de masas con plasma acoplado inductivamente (ICP-MS) fueron usadas para el análisis de las muestras. El análisis por ICP-MS demostró concentraciones importantes de aluminio, azufre, hierro, calcio, magnesio, titanio, metales alcalinos y alcalinotérreos, elementos característicos de una composición andesítica. Mediante el análisis por XRD se determinó que los minerales mayoritarios presentes en la fase cristalina son plagioclasas, ortoclasas y óxidos de silicio (cristobalina), y entre los minoritarios fueron encontrados: óxidos de hierro (magnetita, hematita y maghemita), yeso, dolomita y mayenita. El estudio bibliográfico de las diferentes aplicaciones tanto de los elementos y minerales encontrados en la ceniza, permite recomendar el uso de este material como agregado en materiales y construcción gracias a su composición mineralógica, y evitar su uso como fertilizante sin un análisis previo del $\mathrm{pH}$ del suelo, ya que podría resultar potencialmente tóxico.

\section{ABSTRACT}

This study had as main goal the mineralogical and multielemental analysis of the volcanic ash product of the eruption of the Cotopaxi volcano in August of 2015; in order to determine possible applications and impacts. The X-ray diffraction (XRD) technique and inductively coupled plasma mass spectrometry (ICP-MS) technique were used for the sample analysis. ICP-MS analysis showed important concentrations of aluminum, sulfur, iron, calcium, magnesium, titanium, alkali metals and alkaline-earth elements according of an andesitic composition. Through the analysis by XRD was determined that the major minerals present in the crystalline phase are plagioclases, orthoclases and silicon oxides (cristobalin); and iron oxide (magnetite, hematite and maghemite), gypsum, dolomite and mayenite were found among the minorities compounds. The bibliographic study of the different applications of the elements and minerals found in the ash allows recommending the use of this material as an aggregate in materials and construction due to its mineralogical composition, and avoiding its use as a fertilizer without a previous $\mathrm{pH}$ soil determination as it could be potentially toxic. 


\section{INTRODUCCIÓN}

El volcán Cotopaxi se encuentra ubicado sobre la Cordillera Oriental (Real) de los Andes Ecuatorianos, a una distancia de $35 \mathrm{~km}$ al noreste de Latacunga y $45 \mathrm{~km}$ al sureste de Quito (Latitud 056'06" S; Longitud 78³6’55" O). El Cotopaxi es considerado uno de los volcanes más peligrosos del mundo debido a la frecuencia de sus erupciones, su estilo eruptivo, su relieve, su cobertura glaciar y por la cantidad de poblaciones potencialmente expuestas a sus amenazas; cuenta con una altura de 5897 msnm y un diámetro de 20 km, es un volcán de tipo estratovolcán compuesto y se encuentra en estado activo desde el año 2015 (Aguilera, Toulkeridis \& Dueñas, 2006; Instituto Geofísico Escuela Politécnica Nacional, 2016).

Desde agosto de 2015, el volcán Cotopaxi ha empezado un nuevo proceso eruptivo, aunque hasta el momento no se ha producido una erupción a gran escala. En la actualidad, la actividad superficial observada en el volcán Cotopaxi mantiene bajos niveles, y se ha caracterizado principalmente por la emisión de gases. No se ha observado la presencia de ceniza desde fines de enero de 2016 (Instituto Geofísico EPN, 2016).

La ceniza volcánica es, sin dudarlo, el producto de erupción que cubre un área más amplia y afecta a mayor población. Después de una erupción, las autoridades civiles, los productores agrícolas y la población, tienen a menudo una gran preocupación por los efectos de la ceniza volcánica en la salud, tanto de humanos como de animales, en el abastecimiento de agua potable, los daños en los cultivos y en los suelos.

La ceniza volcánica contiene una gama de elementos potencialmente tóxicos tales como azufre, aluminio, y en ocasiones elementos radiactivos, que pueden ser puestos en libertad, y estarán rápida o lentamente en contacto con el agua (Casadevall, 1991; Stewart, 2013).

La ceniza volcánica está conformada de pequeños fragmentos de roca, minerales y vidrio volcánico (óxido de silicio amorfo). A diferencia de la ceniza suave producida por la quema 
de madera, la ceniza volcánica es dura, abrasiva, e insoluble en agua. En general, las partículas de ceniza volcánica tienen un diámetro aproximado de 2 milímetros o menor. Las partículas gruesas de ceniza volcánica tienen una apariencia similar a granos de arena, mientras que las partículas muy finas son polvo (Casadevall, 1991).

La ceniza es un producto de erupciones volcánicas explosivas, se produce cuando los gases dentro de la cámara de magma (roca fundida) de un volcán se expanden con violencia, empujando el magma hacia arriba y fuera del volcán. La fuerza de estas explosiones rompe y propulsa la roca líquida en el aire, el magma se enfría y solidifica en fragmentos de roca y vidrio volcánico. Las erupciones también pueden romper la roca sólida de la cámara de magma y de las laderas del volcán. Estos fragmentos de roca se pueden mezclar con los fragmentos de lava solidificados en el aire, creando una nube de ceniza. El viento puede transportar las pequeñas partículas de cenizas volcánicas a grandes distancias. Se ha encontrado ceniza a miles de kilómetros de distancia del sitio de la erupción. Cuanto menor sea el tamaño de la partícula, más viajará por acción del viento (Stewart \& Johnston, 2006).

Análisis de la ceniza volcánica en erupciones de otros volcanes, indican que la ceniza está compuesta principalmente de óxidos de silicio, aluminio, hierro y en menor porcentaje de óxidos de metales alcalinos y alcalinotérreos, tales como $\mathrm{CaO}, \mathrm{MgO}$, $\mathrm{Na}_{2} \mathrm{O}, \mathrm{K}_{2} \mathrm{O}$; además, contiene sales solubles como consecuencia de la condensación de ácidos minerales fuertes durante su formación. Esta composición varía dependiendo del tipo de erupción. La ceniza, al entrar en contacto con fuentes de agua, aumenta la concentración de metales como el hierro, manganeso y aluminio. Además, la acidez de la ceniza puede causar reacciones de corrosión de componentes metálicos (Taylor \& Lichte, 1980; Papale, 2014).

La composición química de la ceniza está directamente relacionada con la química del magma que la originó. La composición del magma varía del basalto (bajo en sílice) hasta la riolita (alto en sílice). Los elementos mayores que se analizan para caracterizarla son: silicio, aluminio, titanio, 
hierro, magnesio, calcio, sodio, potasio, cromo, manganeso, fósforo. Los elementos menores son: níquel, cobalto, rubidio, uranio en trazas, torio y tierras raras (Guevara, 2015).

La composición mineralógica de la ceniza volcánica se ve caracterizada por la presencia de silicatos, que pueden dividirse en minerales coloreados e incoloros. Los minerales coloreados contienen considerables cantidades de iones metálicos de transición como el hierro, mientras los incoloros no. Además de los silicatos, se encuentran minerales como óxidos de hierro y titanio en casi todos los magmas. Las fases cristalinas que componen este material son principalmente cuarzo (óxido de silicio) y feldespatos (silicoaluminatos de calcio y magnesio). Óxidos de hierro y de hierro/titanio también pueden estar presentes en varias proporciones, dependiendo de las condiciones de la fuente del magma (Guevara, 2015).

La caracterización de la ceniza volcánica se realiza mediante técnicas instrumentales avanzadas que proporcionan información sobre su composición, las más usadas son: la espectrometría de masas con induc- ción de plasma acoplado (ICP-MS, por sus siglas en inglés) y la difracción de rayos $X$ (XRD, por sus siglas en inglés). La técnica ICP-MS está reconocida como un método rápido y preciso para la determinación de los elementos en general, además, de tierras raras (REEs), metales preciosos y elementos traza en muestras geológicas. Esta técnica permite establecer, de una manera rápida y certera, si la ceniza cuenta con elementos de utilidad o elementos potencialmente tóxicos. Muchos de estos elementos se comportan de manera diferente, según los factores tales como el $\mathrm{pH}$, por lo que es necesario realizar este análisis complementario.

Además, la XRD es la principal técnica usada para identificar y cuantificar los minerales presentes en la ceniza volcánica. Cada mineral o compuesto tiene un patrón de difracción de rayos $\mathrm{X}$ característico, cuya "huella digital" puede ser comparada con una base de datos. El análisis de los datos de difracción de rayos $\mathrm{X}$ es reconocido como el método más potente disponible para el análisis cuantitativo de fase cristalina (Thomas, 2013). 


\section{MATERIALES Y MÉTODOS}

\section{Toma de muestra}

Para el presente estudio, la ceniza se colectó en el sector de Machachi, en una hacienda afectada por la caída de ceniza (Coordenadas: 0³2'43.7" sur, $78^{\circ} 35^{\prime} 54.4^{\prime \prime}$ oeste) ubicada a $24 \mathrm{~km}$ del cráter del Cotopaxi. Se recolectaron cuatro muestras de ceniza de los techos de varias estructuras dentro de la propiedad, que contaban con recubrimientos plásticos, en los cuales se acumuló la ceniza producto de la erupción del volcán Cotopaxi del 14 de agosto del 2015; las muestras se almacenaron en fundas plásticas $\mathrm{Zi}$ ploc ${ }^{\circledR}$ y se guardaron en un lugar protegido del sol y a temperatura ambiente. Todas las muestras se recolectaron el mismo día de la erupción, para evitar contaminación por partículas ajenas como polvo.

\section{Análisis multielemental}

El equipo utilizado en este estudio fue un ICP-MS Agilent modelo 7700e, para este análisis se siguió el método EPA 6020B. Primeramente se realizó la digestión de las muestras mediante digestión abierta. Para esto se pesaron
0,5 gramos de muestra seca en un tubo de ensayo de tapa rosca. Se añadieron 2,3 $\mathrm{mL}$ de $\mathrm{HNO}_{3}$ concentrado y $0,7 \mathrm{~mL}$ de agua tipo I de acuerdo con la ASTM, se calentó la muestra a $95 \pm 5{ }^{\circ} \mathrm{C}$ y se dejó en reflujo durante 1 hora. A la muestra fría se añadió 1 $\mathrm{mL}$ de agua y $0,5 \mathrm{~mL}$ de $\mathrm{H}_{2} \mathrm{O}_{2} 30 \%$, se calentó nuevamente durante 25 minutos para iniciar la reacción del peróxido, se mantuvo el calentamiento hasta que desapareció la efervescencia y se dejó enfriar, luego se añadieron $2,0 \mathrm{~mL}$ de $\mathrm{HCl}$ concentrado, y nuevamente se calentó durante 25 minutos. La solución fría se aforó a un volumen de $50 \mathrm{~mL}$. Las soluciones de las muestras se centrifugaron, filtraron y diluyeron 5x. Esta dilución se analizó directamente en el equipo ICP-MS. En el análisis por ICP-MS se usaron varios modos de celda de colisión (CRC) para el análisis de cada elemento, tales como el modo helio, helio de alta energía o no gas, en función de si existían interferencias específicas para cada elemento. 


\section{Análisis mineralógico}

El equipo utilizado en este estudio fue un difractómetro de Rayos X marca Bruker modelo D2 PHASER. Para la preparación de la muestra se siguió el siguiente procedimiento: se molió 1 gramo de la muestra en un mortero de ágata, posteriormente se pasó por un tamiz malla 100 micras; se colocó cuidadosamente la muestra en el portamuestras aplanando el polvo con una placa de vidrio, y retirando el exceso de polvo; se introdujo el portamuestras en el equipo y se procedió a su análisis cualitativo y cuantitativo.

\section{Análisis complementarios}

Para el análisis potenciométrico se utilizó un potenciómetro marca Metler-Toledo modelo Seven. Se pesaron 20 gramos de suelo en un vaso de precipitación de $50 \mathrm{~mL}$, se añadieron 20 mL de agua y se agitó continuamente la suspensión durante 5 minutos. Se dejó en reposo la suspensión del suelo durante aproximadamente 1 hora para permitir que la mayor parte de la arcilla suspendida precipite, se centrifugó y decantó la fase acuosa para la medición de $\mathrm{pH}$.
El análisis del contenido de óxido de silicio se realizó mediante gravimetría. Se pesaron 0,25 gramos de muestra en un vaso de $300 \mathrm{~mL}$. Se adicionaron $15 \mathrm{~mL}$ de $\mathrm{HCl}$ concentrado, $5 \mathrm{~mL}$ de $\mathrm{HNO}_{3}$ concentrado y $15 \mathrm{~mL}$ de $\mathrm{H}_{2} \mathrm{SO}_{4}$ diluido (1:1). Se calentó lentamente durante 10 minutos para disolver la muestra agitando periódicamente. Se calentó hasta semisequedad y eliminación de humos blancos, se dejó durante 15 minutos más, después de la aparición de los humos blancos. Se dejó enfriar a temperatura ambiente y se adicionaron $50 \mathrm{~mL}$ de agua desionizada, se calentó nuevamente hasta disolver la muestra y se filtró sobre papel filtro lavando el residuo 5 o 6 veces con agua caliente. Se colocó el papel de filtro dentro de un crisol de platino y se lo llevó a la estufa a $105{ }^{\circ} \mathrm{C}$ hasta sequedad. Luego se colocó el crisol en la mufla (temperatura de $450{ }^{\circ} \mathrm{C}$ ), hasta calcinación total a $1000{ }^{\circ} \mathrm{C}$ durante una hora. Una vez frío se pesó el crisol, y se realizaron los cálculos. 


\section{RESULTADOS}

\section{Análisis multielemental}

Se analizaron un total de 69 elementos. La Tabla 1 contiene los resultados generales obtenidos para cada una de las muestras analizadas expresados en $\mathrm{mg} / \mathrm{kg}$ o porcentaje (\%), de acuerdo con la concentración en la que fueron encontrados. La Tabla 1 solo contiene información de los elementos encontrados, no se incluyen los elementos no detectados o encontrados por debajo del límite de cuantificación del método. Los elementos analizados, pero no encontrados en las muestras fueron: antimonio, berilio, bismuto, cadmio, cesio, estaño, erbio, europio, germanio, hafnio, indio, iterbio, lutecio, mercurio, plata, talio, tantalio, teluro, terbio, tulio y wolframio; además de todos los metales preciosos analizados: oro, iridio, osmio, paladio, platino, renio, rodio y rutenio.

\section{Análisis mineralógico}

Se compararon los difractogramas obtenidos con las bibliotecas especializadas utilizando los softwares: EVA para identificar los minerales presentes, y TOPAS para la cuantificación; el equipo tiene un límite de detección aproximado del $1 \%$, por lo que los minerales por debajo de esta concentración no fueron reportados. Los resultados se reportan en base al porcentaje de material cristalino, que fue de $84 \%$ en promedio en todas las muestras. La Tabla 2 contiene información de los minerales presentes en las muestras, su fórmula química y el grupo mineralógico al que pertenecen, junto a los rangos porcentuales encontrados.

\section{Análisis complementarios}

Los análisis complementarios proporcionan información adicional para respaldar y discutir los resultados principales.

Los valores de $\mathrm{pH}$ de las muestras, medidos por triplicado, oscilan entre 4,29-4,58. La determinación del contenido de $\mathrm{SiO} 2$ realizada por duplicado en cada muestra arrojó valores entre $61,93-62,04 \%$ 
Tabla 1. Resultados del análisis multielemental

\begin{tabular}{|c|c|c|c|c|c|}
\hline Elemento & Unidad & Muestra 1 & Muestra 2 & Muestra 3 & Muestra 4 \\
\hline Aluminio & $\mathrm{mg} / \mathrm{kg}$ & 7190,76 & 6917,49 & 6969,33 & 7189,62 \\
\hline Arsénico & $\mathrm{mg} / \mathrm{kg}$ & 0,96 & 1,20 & 1,03 & 0,99 \\
\hline Azufre & $\mathrm{mg} / \mathrm{kg}$ & 8087,01 & 7659,02 & 8271,58 & 8495,81 \\
\hline Bario & $\mathrm{mg} / \mathrm{kg}$ & 22,80 & 23,60 & 22,89 & 23,43 \\
\hline Boro & $\mathrm{mg} / \mathrm{kg}$ & 0,44 & 0,63 & 0,22 & 0,23 \\
\hline Calcio & $\mathrm{mg} / \mathrm{kg}$ & 7990,94 & 8294,10 & 7669,92 & 7959,87 \\
\hline Cerio & $\mathrm{mg} / \mathrm{kg}$ & 8,02 & 8,31 & 7,86 & 8,13 \\
\hline Cromo & $\mathrm{mg} / \mathrm{kg}$ & 5,91 & 6,07 & 5,93 & 6,05 \\
\hline Circonio & $\mathrm{mg} / \mathrm{kg}$ & 3,21 & 3,15 & 3,25 & 3,54 \\
\hline Cobalto & $\mathrm{mg} / \mathrm{kg}$ & 5,27 & 5,39 & 5,21 & 5,45 \\
\hline Cobre & $\mathrm{mg} / \mathrm{kg}$ & 13,24 & 14,4 & 14,87 & 15,23 \\
\hline Disprosio & $\mathrm{mg} / \mathrm{kg}$ & 0,53 & 0,56 & 0,53 & 0,55 \\
\hline Estrocio & $\mathrm{mg} / \mathrm{kg}$ & 62,58 & 59,33 & 60,98 & 62,37 \\
\hline Galodineo & $\mathrm{mg} / \mathrm{kg}$ & 0,77 & 0,80 & 0,73 & 0,77 \\
\hline Galio & $\mathrm{mg} / \mathrm{kg}$ & 2,15 & 2,17 & 2,03 & 2,13 \\
\hline Hierro & $\%$ & 1,35 & 1,35 & 1,33 & 1,38 \\
\hline Lantano & $\mathrm{mg} / \mathrm{kg}$ & 3,61 & 3,70 & 3,50 & 3,54 \\
\hline Litio & $\mathrm{mg} / \mathrm{kg}$ & 0,97 & 1,06 & 0,91 & 0,91 \\
\hline Magnesio & $\mathrm{mg} / \mathrm{kg}$ & 115,00 & 116,00 & 108,00 & 112,00 \\
\hline Manganeso & $\mathrm{mg} / \mathrm{kg}$ & 64,48 & 68,01 & 63,64 & 66,23 \\
\hline Molibdeno & $\mathrm{mg} / \mathrm{kg}$ & 0,55 & 0,44 & 0,75 & 0,77 \\
\hline Neodimo & $\mathrm{mg} / \mathrm{kg}$ & 4,46 & 4,57 & 4,32 & 4,47 \\
\hline Niquel & $\mathrm{mg} / \mathrm{kg}$ & 3,32 & 3,52 & 3,17 & 3,10 \\
\hline Niobio & $\mathrm{mg} / \mathrm{kg}$ & 0,94 & 0,42 & 0,70 & 0,65 \\
\hline Fósforo & $\%$ & 0,035 & 0,037 & 0,036 & 0,036 \\
\hline Potasio & $\%$ & 0,035 & 0,040 & 0,034 & 0,036 \\
\hline Praseodimio & $\mathrm{mg} / \mathrm{kg}$ & 1,05 & 1,10 & 1,06 & 1,05 \\
\hline Rubidio & $\mathrm{mg} / \mathrm{kg}$ & 0,78 & 0,77 & 0,78 & 0,77 \\
\hline Samario & $\mathrm{mg} / \mathrm{kg}$ & 0,93 & 0,96 & 0,87 & 0,91 \\
\hline Selenio & $\mathrm{mg} / \mathrm{kg}$ & 0,72 & 0,68 & 0,62 & 0,64 \\
\hline Sodio & $\%$ & 0,125 & 0,132 & 0,119 & 0,124 \\
\hline Plomo & $\mathrm{mg} / \mathrm{kg}$ & 1,03 & 1,09 & 1,12 & 1,11 \\
\hline Torio & $\mathrm{mg} / \mathrm{kg}$ & 0,65 & 0,65 & 0,55 & 0,55 \\
\hline Titanio & $\mathrm{mg} / \mathrm{kg}$ & 811,76 & 767,94 & 807,47 & 839,95 \\
\hline Uranio & $\mathrm{mg} / \mathrm{kg}$ & 0,18 & 0,19 & 0,18 & 0,17 \\
\hline Vanadio & $\mathrm{mg} / \mathrm{kg}$ & 67,27 & 66,20 & 67,27 & 69,10 \\
\hline Zinc & $\mathrm{mg} / \mathrm{kg}$ & 15,68 & 15,43 & 15,25 & 16,00 \\
\hline Escandio & $\mathrm{mg} / \mathrm{kg}$ & 1,31 & 1,58 & 2,68 & 2,40 \\
\hline Itrio & $\mathrm{mg} / \mathrm{kg}$ & 2,86 & 2,56 & 2,76 & 2,72 \\
\hline
\end{tabular}


Tabla 2. Resultados del análisis mineralógico

\begin{tabular}{|c|c|c|c|}
\hline Mineral & Fórmula química & Grupo Mineralógico & $\%$ en muestras \\
\hline Yeso & $\mathrm{CaSO}_{4}$ & Sulfato & $4,36-6,50$ \\
\hline Hematita & $\mathrm{Fe}_{2} \mathrm{O}_{3}$ & \multirow{3}{*}{ Óxido de hierro } & \multirow{3}{*}{$5,29-9,89$} \\
\hline Magnetita & $\mathrm{Fe}_{3} \mathrm{O}_{4}$ & & \\
\hline Maghemita & $\mathrm{Fe}_{2} \mathrm{O}_{3}$ & & \\
\hline Albita & $\mathrm{NaAlSi} 3 \mathrm{O}_{8}$ & & \multirow{8}{*}{$52,66-60,94$} \\
\hline \multirow{3}{*}{ Andesita } & $(\mathrm{Na}, \mathrm{Ca}) \mathrm{Al} \mathrm{AlSi} 3 \mathrm{O}_{8}$ & & \\
\hline & $(70 \%$ a $50 \%)$ de $\mathrm{Na}$ & & \\
\hline & en relación Na/Ca & Feldespato & \\
\hline \multirow{3}{*}{ Bytownita } & $(\mathrm{Na}, \mathrm{Ca}) \mathrm{Al} \mathrm{AlSi} 3 \mathrm{O}_{8}$ & de Plagioclasa & \\
\hline & (30\% a 10\%) de $\mathrm{Na}$ & & \\
\hline & en relación Na/Ca & & \\
\hline Anortina & $\mathrm{Ca} \mathrm{Al} 2 \mathrm{Si}_{3} \mathrm{O}_{8}$ & & \\
\hline Ortoclasa & $\mathrm{KAISi} i \mathrm{O}_{8}$ & Feldespato de Potasio & $11,31-14,75$ \\
\hline Mayenita & $\mathrm{Ca}_{2} \mathrm{Al}_{14} \mathrm{O}_{33}$ & $\begin{array}{c}\text { Óxido de Calcio } \\
\text { y aluminio }\end{array}$ & $0,87-2,40$ \\
\hline Cristobalina & $\mathrm{SiO}_{2}$ & Óxido de silicio & $9,24-18,63$ \\
\hline Dolomita & $\mathrm{CaMg}\left(\mathrm{CO}_{3}\right)_{2}$ & Carbonato & $0,00-3,16$ \\
\hline
\end{tabular}

\section{DISCUSIÓN}

\section{Efectos del pH}

El pH de las muestras de ceniza analizadas se encuentra entre 4,30 4,57 , en este caso, el uso directo de la ceniza como fertilizante no es recomendable, ya que el $\mathrm{pH}$ de este material es muy bajo; lo cual podría acarrear consecuencias negativas para los cultivos, pues la mayoría de los procesos microbianos, incluyendo la descomposición de la materia orgánica y el ciclo de los nutrientes, se reducen en un suelo ácido, porque el crecimiento y la reproducción de los microorganismos del suelo, principalmente bacterias y hongos, se reducen (Gazey, 2016).

Otros efecto de un pH bajo, es la diminución de la disponibilidad de los principales nutrientes de las plantas. 
Esto a menudo se produce en suelos ácidos, porque nutrientes como el nitrógeno, fósforo, potasio, azufre, calcio y magnesio, están menos disponibles para las plantas (Gazey, 2016).

\section{Análisis multielemental}

Elementos tales como hierro $(1,33-$ 1,38 \%), calcio (7670 - $8294 \mathrm{mg} / \mathrm{kg})$, aluminio (6917 - $7191 \mathrm{mg} / \mathrm{kg})$, titanio (768 - $840 \mathrm{mg} / \mathrm{kg})$, sodio $(0,119-$ $0,132 \%)$, potasio $(0,034-0,040 \%)$ y magnesio (108 - $116 \mathrm{mg} / \mathrm{km})$ se encuentran en concentraciones importantes en las muestras de ceniza analizadas, lo cual es de esperarse ya que estos elementos son de gran abundancia en la corteza terrestre y concuerda con los elementos predominantes reportados en otros estudios (Casadevall, 1991; Stewart, 2013). A pesar de las concentraciones de estos elementos, no es posible extraerlos de este material, ya que no se encuentran en concentraciones rentables (Emsley, 2011).

Debido al proceso de formación del magma que se da por procesos de subducción de las placas de la corteza terrestre, los elementos poco abundantes en la corteza terrestre, tales como metales preciosos y tierras raras, no se encuentran en el magma que da origen a la ceniza volcánica, esto explica las concentraciones nulas de dichos elementos en este material (Gazey, 2016).

El azufre se encuentra en gran concentración (7659 - 8496 mg/kg), supera así los límites de concentración establecidos por el Acuerdo Ministerial 097 (250 mg/kg), documento que regula los criterios de calidad ambiental que deben cumplir los suelos en Ecuador. La alta concentración de este elemento supone un riesgo para los cultivos, ya que puede ser tóxico y generar compuestos que pueden alterar el pH del suelo (Emsley, 2011).

La ceniza volcánica analizada tiene una elevada concentración de aluminio (6917 - $7191 \mathrm{mg} / \mathrm{kg}$ ), lo cual la convertiría en un agente tóxico para los cultivos, si se tiene en cuenta que el $\mathrm{pH}$ del suelo bajaría a un valor inferior a 5. Los niveles tóxicos de aluminio en la solución del suelo afectan a la división celular de la raíz y la capacidad de la raíz de alargarse, reducen el crecimiento de las raíces y su ramificación. Esto se traduce en una mala cosecha y crecimiento de los 
pastos. Los efectos de la toxicidad del aluminio son más notables en las temporadas secas. Las raíces son incapaces de crecer de manera efectiva a través del suelo subsuperficial ácido, que forma una barrera y restringe el acceso al agua del subsuelo, afectando el rendimiento de los cultivos (Gazey, 2016).

\section{Análisis mineralógico}

El resultado de la determinación del contenido de $\mathrm{SiO}_{2}$ en las muestras se encuentra alrededor del $62 \%$, que comprende el rango de composición de las andesitas, por lo que se puede clasificar el magma producto de esta erupción como magma andesítico, que es un magma de consistencia espesa, que forma flujos piroclásticos que dan origen a estratovolcanes (conos volcánicos formados por estratos de material), esto es concordante con el típico evento eruptivo andesítico, característico de estratovolcanes como el Cotopaxi (Guevara, 2015). Desde el punto de vista mineralógico, las 4 muestras de ceniza son relativamente homogéneas, con un conjunto de minerales compuestos principalmente por plagioclasas entre un 53 $61 \%$, seguido de ortoclasa entre un 11 - $15 \%$, óxido de silicio (cristobalina) entre un 9 - $19 \%$, óxidos de hierro (magnetita, hematita y maghemita) entre un $5-10 \%$, yeso entre un $4-7$ $\%$, dolomita entre un 0 - $3 \%$ y mayenita $1-2 \%$.

En base el grado de cristalinidad de 82 a $86 \%$, se puede decir que la erupción de agosto de 2015, que originó la ceniza, se trató de una explosión entre freática (expulsión de vapor de agua y gases) y freatomagmática (expulsión de vapor de agua, gases y magma), ya que en una erupción freática la roca madre del edificio volcánico es arrastrada, y por esta razón se puede apreciar que la mayoría de minerales son de tipo no magmáticos como: plagioclasas, cristobalina, minerales de hierro entre otros (Tsukasa \& Mitsuhiro, 2002). 


\section{CONCLUSIONES}

La composición elemental de los depósitos de ceniza del volcán Cotopaxi de agosto de 2015 muestra concentraciones importantes de aluminio, azufre, hierro, calcio, magnesio y titanio.

Las muestras de ceniza del volcán Cotopaxi analizadas, presentan una composición mineralógica compuesta principalmente por plagioclasas entre un 53 - 61\%, seguido de ortoclasa entre un 11 - $15 \%$, óxidos de silicio (cristobalina) entre un 9 - $19 \%$, óxidos de hierro (magnetita, hematita y maghemita) entre un 5 - $10 \%$, yeso entre un 4 - 7\%, dolomita entre un 0 a $3 \%$ y mayenita 1 a $2 \%$.

En base al grado de cristalinidad, se puede decir que se trató de una explosión entre freática y freatomagmática, lo cual justifica la presencia de minerales de tipo no magmáticos.

Las muestras analizadas, presentan un contenido de $\mathrm{SiO}_{2}$ correspondiente al rango de las andesitas, lo cual clasifica la erupción de agosto del 2015 del Cotopaxi como una erupción de tipo andésica.

La composición elemental muestra que este material no posee elementos tóxicos tales como metales pesados, aunque según el $\mathrm{pH}$ del suelo donde se depositen los elementos que se encuentran en altas concentraciones, como el aluminio y el azufre, podrían ser tóxicos. Por su alta acidez (4,3 a $4,6 \mathrm{pH}$ ) no se recomienda utilizar este material como fertilizante sin un análisis de $\mathrm{pH}$ del suelo, ya que podría ser potencialmente tóxico para los cultivos.

La composición mineralógica de este material muestra que contiene altos porcentajes de feldespatos, lo cual es de considerar para proyectar su uso como agregado para la elaboración de hormigón y asfalto. 


\section{LISTA DE REFERENCIAS}

Aguilera, O., Toulkeridis, T. \& Dueñas, W. (2006). El volcán Cotopaxi, una amenaza que acecha: un enfoque a la gestión del riesgo volcánico en el Ecuador: Proyecto PREVOLCO "Prevención de los Riesgos Asociados con la Erupción de Volcán Cotopaxi". Quito: Fundación FOES.

Casadevall, T. (1991). Volcanic ash and aviation safety: Proceedings of the First International Symposium on volcanic ash and Aviation safety. Seattle, USA.: U.S Geological Survey Bulletin 2047.

Emsley, J. (2011), Natures Building Blocks, First edition, Oxford, UK: Oxford University Press.

Gazey, C. (2016), Effects of soil acidity, Recuperado de https://www.agric.wa.gov.au/soil acidity/effects-soil-acidity?page=0\%2C2 (20 de diciembre de 2016)

Guevara, A. (2015). Estudio de las propiedades de las cenizas del volcán Tungurahua, Ecuador, para identificar los factores que determinan su impacto en suelos y cultivos. Universidad Católica de Lovaina, Lovaina, Bélgica.

Instituto Geofísico EPN. (2016). Actualización de la actividad eruptiva--volcán Cotopaxi $\mathrm{n}^{\circ} 16$ y 17. Quito: Instituto Geofísico EPN.

Papale, P. (2014). Volcanic Hazards, Risks and Disasters. Rome, Italy: Elsevier.

Stewart, C. (2013). Protocol for analysis of volcanic ash samples for assessment of hazards from leachable elements. New Zealand: Massey University.

Stewart, D. y Johnston, B. (2006). Contamination of water supplies by volcanic ash fall: A literature review and simple impact modelling Honorary Auckland. New Zealand: School of Geography and Environmental Sciences, University of Auckland.

Taylor, H. y Lichte, F. (1980). Chemical composition of Mount St. Helens volcanic ash. Denver, USA: Geophysical Research Letters 7(11). 
Thomas, R. (2013). Practical Guide to ICP-MS: A Tutorial for Beginners. Third Edition. Florida, USA: CRC Press.

Tsukasa, O. y Mitsuhiro, N. (2002). Minerals in volcanic Ash: Non -magmatic minerals. Japan: Department of mineralogy, Tohoku University.

U. S. Geological Survey Open-File Report 01-04 (2016), A Laboratory Manual for X-Ray Powder Diffraction: X-Ray Diffraction Primer, USA: Geological survey. 
